\title{
Research on Posture Comfort Degree Based on Biomechanics and Its Application
}

\author{
Rui Liang \\ Herbin Institute of Technology, Harbin 150001, China. \\ 410488717@qq.com
}

Keywords: biomechanics; subjective evaluation; simulation experiment; entity experiment; posture comfort degree.

\begin{abstract}
When working or exercising, the human body is coordinated by its movement feedback system, which always coordinates various parts of the body to a high level as much as possible. Research on posture comfort degree based on biomechanics can make the results of adjustment intuitive and make the description of posture comfort degree objective. In this paper, the author will expound three research methods of posture comfort degree based on biomechanics, that is, subjective evaluation method, simulation experiment method and entity experiment method, discuss the advantages and disadvantages of the above three methods, find the problems in research period, and suggest to apply the research results to products design in other fields to improve the competitiveness of other products in the market.
\end{abstract}

\section{Introduction}

Posture comfort degree refers to the matching degree between the posture of a person when operating a device and the operator's physiological characteristics. The posture comfort degree will directly affect the accuracy and performance when operating the device, and will affect the workload, degree of fatigue, and even the health and safety of people; therefore, the evaluation of posture comfort degree plays an important role in design and optimization of products. At present, the research methods of posture comfort degree based on biomechanics include subjective evaluation method, simulation experiment method and entity experiment method. In this paper, the author will expound the present situation and the application of posture comfort degree based on biomechanics from the above three aspects.

\section{Research Methods of Posture Comfort Degree Based on Biomechanics}

Subjective Evaluation Method. Subjective evaluation method is the most commonly used experiment method. As early as 1963, A. Wisner and R. Rebiffe ${ }^{[1]}$ researched the human fatigue and comfort degree related to human posture based on human anatomy and physiology. In 1998, in order to find the most comfortable driving posture, Porter et al. ${ }^{[2]}$ invited a large number of subjects to describe the posture comfort degree with subjective evaluation method and obtained the corresponding relationship between driving comfort degree and the angle of the joints through analysis. This experiment method is easy to implement, but it requires a large number of subjects to conduct subjective description. The cognitive level, feelings, emotions and other individual differences of subjects will have an impact on the experiment results, so this method is time-consuming with poor repeatability.

Simulation Experiment Method. With the development of science and technology, simulation experiment method can be used to analyze the posture comfort degree through analyzing human muscle, pressure distribution and joint torque ${ }^{[3]}$. With repeatability, low cost, high efficiency, and small error, this experiment method can provide basis for parameterization design of man-machine engineering. At present, simulation experiment method has received more and more attention. 
Research on Posture Comfort Degree Based on Human Muscle. The muscle fatigue degree of the operators is one of the important indexes to evaluate the posture comfort degree. In China, Chen Jinghui et al. ${ }^{[4]}$ found that the evaluation of driver's static posture comfort degree includes evaluation of driving posture and muscle load. In the course of experiment, the muscle load cannot be measured and calculated; however, the load of relative joints can reflect the muscle load, and the joint load can be calculated with method of inverse kinematics, so the evaluation model of posture comfort degree which was based on joint load and used for layout and optimization design was constructed. Yang Feng ${ }^{[5]}$ from Hunan University researched the relationship between the design parameters of man-machine interface in the car cab and muscle fatigue, which provided basis for parameterization design of man-machine engineering. Li Xiaona et al. ${ }^{6]}$ adopted evaluation criteria of muscle fatigue and provided an effective evaluation method for design of comfort degree of bikes.

In foreign countries, Hardeep S. Ryait et al. ${ }^{[7]}$ measured the entropy-based electromyography (EMG) signal of different parts of the human body in different working conditions and compared the comfort degree of EMG signal in the working state. The reason for choosing upper limb for analysis is that the upper limb is conducive to record the EMG signal, and its joint movement space is large.

Research on Posture Comfort Degree Based on Pressure Distribution. The researches of posture comfort degree based on pressure distribution are mainly used for man-machine engineering designs of footwear products, tool handles, and device to be used in sitting or lying posture. Hong Youlian et al. ${ }^{[8]}$ concluded that laced running shoes play better effect in fixing and protecting the feet through comprehensive comparison of subjective comfort degree, plantar pressure and hind foot movement parameters. In 2005, Yong-Ku Kong et al. conducted grab measurement of the handles with diameter of 20-50 mm with Data Glove, and concluded that among the diameter of 35-45 mm, the comfort degree of grab postures is the highest ${ }^{[9]}$. Yang Dewei ${ }^{[10]}$ from Zhejiang University simulated the pressure of hands when grabbling handles through software, providing a scientific basis for further design of the handle shape.

When using products in sitting or lying posture, the contact surface between user and the product is large, so is the influence of pressure distribution on evaluation of posture comfort degree. Li Zhelin et al. ${ }^{[11]}$ from School of Design of South China University of Technology measured and analyzed the pressure intensity and contact area of the bathtub imposed by users, and optimized the design of bath surface, which has effectively improved the comfort degree of users. Siefert et al. ${ }^{[12]}$ researched the effect of static body pressure distribution of auto seats on the comfort degree of auto seats.

Research on Posture Comfort Degree Based on Joint Torque. At present, the research of posture comfort degree based on joint torque is mainly used for layout design and optimization of cabins with complex interior structure, such as cabin of aircraft, car, and bathyscaphe. Workers in these cabinets need to get information of the instrument and outside world relying on vision, auditory and tactile sense, make quick judgments, and finally implement correct operation through hands, feet and other locomotive organs ${ }^{[13]}$. Therefore, the posture comfort degree of workers is an important factor in evaluating cabin layout.

Deng $\mathrm{Li}$ et al. introduced joint torque into the evaluation of joint comfort degree, solving the problem of overall comfort assessment of upper limbs when combined with different joint angles ${ }^{\text {[14] }}$. Yin Qingsong et al. ${ }^{[15]}$ introduced joint torque into the analysis of the force and comfort degree of the lower limb joint when driving a car, which quantitatively reflected the joint comfort degree and provided reference for the design of the cabin. Chen Zi'ang ${ }^{[16]}$ from Hefei University of Technology introduced joint torque into researches of posture comfort degree when driver turns the steering wheel or stepping on the pedal, providing a reference for optimized layout of cabin. People cannot get the joint torque through direct measurement; therefore, it is necessary to calculate the required joint torque by establishing the model of kinematics in the simulation software, and then establish the posture comfort model based on the joint torque.

Entity Experiment Method. Entity experiment method can be used to collect data in action capture system, pressure distribution measurement system and other man-machine performance experimental equipment through making prototype or using real machine ${ }^{[17]}$. The experimental results obtained with entity experiment method are objective and accurate; however, the implementation of the 
experiment requires a lot of equipment, long experimental process and high experiment cost; moreover, this method cannot be used in the early stage of the design process. With the development of CAE technology, more and more people begin to use simulation experiment method to evaluate posture comfort degree.

\section{Conclusions}

In summary, the conclusions obtained from the research on posture comfort degree based on biomechanics are relatively objective and the applications of which to the product design and cabin layout are also increasing. However, there are still problems to be solved in the course of the research. Compared with the population of China, the number of subjects in all experiments is very small, which may lead to a large deviation in the experimental results.

Due to problems in the subjective evaluation method and the entity experiment method, the most commonly used method in the research on posture comfort degree based on biomechanics was the simulation experiment method. The research on posture comfort degree based on pressure distribution is applied in the design optimization of footwear products, tool handles, and products with large contact area with users, such as car seats, bathtubs, seat backs, etc. The research on posture comfort degree based on human muscle and the research on posture comfort degree based on joint torque focused on the layout optimization of complex cabin, and the simulation experiment method is rarely used in analysis on posture comfort degree of design and production of popular products and machines. Having a good way of man-machine interaction can improve working efficiency and accuracy of products and reduce the fatigue of the users, thereby enhancing the market competitiveness of products. Therefore, research on posture comfort degree based on biomechanics should be gradually applied to the design and production of popular products.

\section{References}

[1] Wisner A, Rebiffe R. Methods of Improving Work-place Layout. The International Journal of Production Research, 1963, 2):1 5-167.

[2] Poter M J, Gyi D E.. Exploring the Optimum Posture for Driver Comfort. International Journal of Vehicle Design（S0143-3369）, 1998, 19 (3) : 255-266.

[3] Zhou Weiwei. Research on Handling Comfort Measurement Method and Optimization Technology in Man-Machine System. Hefei University of Technology, 2012.

[4] Chen Jinghui, Ren Jindong, Lu Shanbin, et al. A Research on the Evaluation of Driver's Posture Comfort. Automotive Engineering, 2013, 35(6):548-552.

[5] Yang Feng. Research on the Automobile Cab Ergonomic Design Based on Biomechanics. Hunan University, 2015.

[6] Li Xiaona, Xiang Zhongxia, et al. Simulation and study of the rider-bicycle system based on Biomechanics. Journal of Machine Design, 2013, 30(9):101-104.

[7] Ryait H S , Agarwal R. Interpretations of Wrist/Grip Operations From SEMG Signals at Different Locations on Arm. IEEE Transactions on Biomedical Circuits \& Systems，2010，4 (2): 101-111.

[8] Hong Youlian, Wang Lin, Zhou Ji, et al. Planter Pressure, Comfort and Rearfoot Motion during Running: Comparison between Laced Running Shoes and Elastic-covered Running Shoes. Chinese Journal of Sports Medicine, 2011, 30(7):613-617.

[9] Yong-Ku Kong, Brian D. Lowe. Evaluation of handle diameters and orientations in a maximum torque task. International Journal of Industrial Ergonomics, 2005, 35: 1073-1084. 
[10] Yang Dewei. The Research on the Three Dimensional Finite Element Model of Grasp-Hand Based On the Analysis of Grasping Hand-Held Tool Handel. Hangzhou: Zhejiang University of Technology, 2012

[11] Li Zhelin, Jiang Lijun, Huang Yurun, et al. Optimal Design of Bathtub Surface Based on Tekscan Pressure Measurement. Journal of Graphics, 2017, 38(1):28-33.

[12] Siefert A, Pankoke S. Wolfel H P. Virtual optimisation of car passenger seats: simulation of static and dynamic effects on drivers' seating comfort. International Journal of Industrial Ergonomics, 2008, 38(5-6): 410.

[13] Zong Licheng, Yu Suihuai, Sun Jinbo, et al. Study on Cabin Layout Optimization with Fish Algorithm. Mechanical Science and Technology for Aerospace Engineering, 2014, 33(2):257-262.

[14] Deng Li, Chen Bo, Yu Suihuai, et al. Research on Evaluation Method of Upper Limb Operating Comfort for Cabin Layout Optimization. Mechanical Science and Technology for Aerospace Engineering, 2017, 36(1):108-113.

[15] Yin Qingsong, Liao Qianfang, Zhou Qianxiang, et al. Analysis of Limb Joint Torque and Comfort in Driving Posture Based On JACK. Space Medicine \& Medical Engineering, 2016, 29(6):440-445.

[16] Chen Ziang. Research on the Automotive Handling Discomfort Based on Joint Torque. Hefei University of Technology, 2016.

[17] Kee D, Lee I, Relationships between subjective and objective measures in assessing postural stresses, Applied Ergonomics, 2012.43 (2) : 277-282 